was determined by a Random Forest to define the relative importance of variables for disease prediction.

Results: AdaptiveNet predicted active disease defined as DAS28-BSR $>2.6$ at the next visit with an overall accuracy of $75.6 \%$ (SD +- $0.7 \%$ ) and a sensitivity and specificity of $84.2 \%$ (SD +- $1.6 \%$ ) and $61.5 \%$ (SD +- $3.6 \%$ ), respectively. The performance of the prediction for correct disease status was significantly higher in patients with a disease duration $>3$ years and positive rheumatoid factor. Regression allowed forecasting individual DAS28-BSR values with a Mean Squared Error (MSE) of 0.9 (SD +- 0.05). Compared to Linear Regression, Random Forests and Support Vector Machines, AdaptiveNet showed an increased performance of $7 \%$ in MSE. MSE was significantly lower in patients with disease duration $>3$ years and with positive anti-CCP antibodies. Feature importance identified number of painful joints, longer disease duration and age as most relevant factors for prediction of remission, whereas medication played a smaller role.

Conclusion: Predictability of disease activity in RA by this deep neural network was stronger in patients with a longer disease history and a positive auto-antibody status, potentially due to a more stable disease course. Generally, AdaptiveNet had a superior capacity to predict numeric RA disease activity compared to classical machine learning architectures, however all investigated models had limitations in low specificity.

REFERENCES:

[1] Hügle M, Kalweit G, Hügle T, Boedecker J. Dynamic Deep Neural Network For Multimodal Clinical Data Analysis. Stud Comput Intell: Springer Verl. 2020.

Acknowledgements: We thank all rheumatologists and their patients for participation to SCQM.The entire SCQM staff was instrumental for data management and support.

A list of rheumatology practices and hospitals that are contributing to the SCQM registries can be found on http://www.scqm.ch/institutions.

Disclosure of Interests: None declared

DOI: 10.1136/annrheumdis-2021-eular.2370

\section{POS0469 \\ FALL RISK ASSESSMENT IN RHEUMATOID ARTHRITIS PATIENTS}

O. Jomaa ${ }^{1}$, H. Migaw ${ }^{2}$, I. Loubiri², M. Slama ${ }^{2}$, S. Zrour ${ }^{1}$, I. Bejia ${ }^{1}$, M. Touzi ${ }^{1}$, J. Mahbouba', N. Bergaoui ${ }^{1} .{ }^{1}$ University Hospital Fattouma Bourguiba Monastir, TUNISIA, Rheumatology Department, Monastir, Tunisia; ${ }^{2}$ University Hospital Fattouma Bourguiba Monastir, TUNISIA, Physical Medicine and Functional Rehabilitation Department, Monastir, Tunisia

Background: Rheumatoid arthritis patients may have an increased risk of falls due to changes caused by the disease such as muscle weakness, joint impairment, reduced mobility and postural instability.

Objectives: The aim of this study was to analyze the occurrence of falls in RA patients and its risk factors.

Methods: Between January2020 and July 2020, 51 patients with RA were included in the study. fall history, and the number of falls within the past 12 month were questioned. All participants were assessed with Timed Up and Go Test (TUGT), One-Leg Stand Test (OLST), Walking and Talking Test (WTT), Sternal Push Test (SPT), Tinetti Test (TT), Four Test Balance Scale (FTBS), The Short Falls Efficacy Scale-International (FES-I), Pain Severity and Patient Global Assessment (PGA) by Visual Analog Scale (VAS), disability by the Health Assessment Questionnaire (HAQ), and disease activity by Disease Activity Score in 28 joints (DAS28) were evaluated in for for each patient. The Kolmogorov-Smirnov, Mann-Whitney and chi-square tests were performed with a significance level of $\mathrm{P} \leq 0.05$.

Results: 22 patients had at least one or more falls. The average age was $54.9 \pm 11.5$ years with a female predominance $($ sex ratio $=0.13)$. Comparing the two groups of patients: those with a history of falls and those without, patients with previous falls were mainly married women $(p=0.57)$, with a low intellectual level $(p=0.63)$. The body mass index in this group was higher $(p=0.01)$ with respectively a higher number of painful and swollen joints $(p=0.16, p=0.07)$; the mean HAQ was higher $(p=0.03)$, and the mean VAS was more important $(p=0.05) .12 / 22$ patients had co-morbidities and were poly-medicated.20/22 had joint feet deformities (hallux valgus was the most common deformity). $21 / 22$ were on low dosage corticosteroid therapy. The mean TUGT was 18.5 seconds ( $>14$ seconds). The OLST was less than 5 seconds in $12 / 22$, the sternal push test was positive in $9 / 22$, WTT was positive in $6 / 22$ and the mean tinetti test was $22.4 \pm 5.4$ suggesting a high risk of falls. The average Short FES-I was $14.6 \pm 4.4$ reflecting a high fear of falls in this group of patients.

Conclusion: Knowledge about risk factors can help to identify high-risk patients in order to decrease their risk of falling, thus preventing fall-related injuries.

DOI: 10.12659/MSM.921862

Disclosure of Interests: None declared

DOI: 10.1136/annrheumdis-2021-eular.2395

\section{POS0470 \\ GLUCOCORTICOID DISCONTINUATION IN EARLY RHEUMATOID AND UNDIFFERENTIATED ARTHRITIS PATIENTS. A SUB-ANALYSIS OF THE BeSt AND IMPROVED STUDIES}

J. M. Maassen ${ }^{1}$, R. Dos-Santos ${ }^{2}$, S. A. Bergstra ${ }^{1}$, R. Goekoop ${ }^{3}$, T. Huizinga ${ }^{1}$, C. Allaart ${ }^{1} .{ }^{1}$ Leiden University Medical Center (LUMC), Rheumatology, Leiden, Netherlands; ${ }^{2}$ Clinical University Hospital, Rheumatology, Santiago de Compostela, Spain; ${ }^{3} \mathrm{Haga}$ Hospital, Rheumatology, The Hague, Netherlands

Background: Discontinuation of glucocorticoids (GC) as bridging therapy in rheumatoid arthritis (RA) treatment is recommended as rapidly as clinically feasible. Little is known about the rate of, and possible characteristics associated with successful GC discontinuation.

Objectives: To evaluate the success rate of GC discontinuation, and to study which factors are associated with successful GC discontinuation.

Methods: Data from two treat-to-target studies; BeSt (target DAS $\leq 2.4$, LDA) and IMPROVED (target DAS $<1.6$, remission) were evaluated for all patients initially treated with a tapered high-dose of oral GC with conventional DMARD(s) GCs were discontinued when DAS $\leq 2.4$ was maintained for 28 weeks in BeSt and as soon as DAS was $<1.6$ in IMPROVED. GC discontinuation could be attempted twice: first attempt called 'primary', second attempt after GC restart called 'secondary'. Discontinuation was considered successful if the treatment target was maintained at the next visit. Univariable, and exploratory forward and backward multivariable logistic regression analyses were performed to identify potential characteristics associated with successful discontinuation ( $p$-value $<0.2$ included in final model).

Results: From the 131 patients initiating combination therapy with prednisone in the BeSt study, 93 attempted discontinuation. Primary discontinuation was successful in 60\% (56/93) and secondary discontinuation in 54\% (19/35). A lower DAS at the visit prior to GC discontinuation and ACPA negativity were associated with successful discontinuation (table 1). Of the 610 patients in the IMPROVED, 400 attempted discontinuation. Primary discontinuation was successful in $61 \%$ $(242 / 400)$, and secondary in $51 \%(71 / 139)$. A lower DAS both at baseline and at the visit prior to GC discontinuation were associated with successful discontinuation (table 1)

Conclusion: Primary GCs discontinuation was successful in approximately $60 \%$ and secondary in $50 \%$ of patients, independent of the treatment target and associated threshold for GC discontinuation. Most baseline characteristics were no predictive of successful GC discontinuation, but ACPA negativity (only in BeSt) baseline DAS (only in IMPROVED) and in both studies DAS prior to GC discontinuation were predictive for successful discontinuation. Based on this data it seems that 'standard' baseline characteristics are insufficient to 'personalize' the duration of temporary GC bridging but the DAS at the moment of GC discontinuation might give guidance.

Table 1. Results logistic regression analyses

\begin{tabular}{|c|c|c|c|c|}
\hline & Univariable & & $\begin{array}{l}\text { Multivariable }^{\mathrm{a}} \\
\mathrm{R}^{2}=0.173\end{array}$ & \\
\hline BeSt & OR $(95 \% \mathrm{Cl})$ & $p$-value & OR $(95 \% \mathrm{Cl})$ & $p$-value \\
\hline Age, year & $1.00(0.98 ; 1.03)$ & 0.98 & & \\
\hline Gender, female & $0.51(0.24 ; 1.09)$ & 0.08 & & \\
\hline Symptom duration $\mathrm{BL}$, weeks & $1.00(0.99 ; 1.01)$ & 0.61 & & \\
\hline $\mathrm{DAS}$ at $\mathrm{BL}$ & $0.92(0.61 ; 1.40)$ & 0.70 & & \\
\hline DAS prior to discontinuation & $0.13(0.05 ; 0.33)$ & $<0.01$ & $0.11(0.04 ; 0.30)$ & $<0.01$ \\
\hline RF, positive & $1.28(0.62 ; 2.69)$ & 0.50 & $2.24(0.81 ; 6,17)$ & 0.12 \\
\hline ACPA, positive & $0.70(0.34 ; 1.43)$ & 0.32 & $0.32(0.12 ; 0.86)$ & 0.02 \\
\hline \multirow[t]{3}{*}{ Erosions, present at BL } & $0.65(0.28 ; 1.49)$ & 0.31 & & \\
\hline & Univariable & & Multivariable $^{a}$ & \\
\hline & & & $R^{2}=0.065$ & \\
\hline IMPROVED & OR (95\% Cl) & p-value & OR (95\% Cl) & p-value \\
\hline Age, year & $1.00(0.99 ; 1.02)$ & 0.64 & & \\
\hline Gender, female & $0.62(0.43 ; 0.89)$ & 0.01 & $0.75(0.51 ; 1.11)$ & 0.15 \\
\hline Symptom duration $\mathrm{BL}$, weeks & $1.00(0.99 ; 1.00)$ & 0.43 & $0.99(0.99 ; 1.00)$ & 0.08 \\
\hline $\mathrm{DAS}$ at $\mathrm{BL}$ & $0.80(0.65 ; 0.98)$ & 0.03 & $0.78(0.62 ; 0.98)$ & 0.03 \\
\hline DAS prior to discontinuation & $0.24(0.15 ; 0.38)$ & $<0.01$ & $0.24(0.14 ; 0.40)$ & $<0.01$ \\
\hline RF, positive & $0.82(0.57 ; 1.17)$ & 0.27 & & \\
\hline ACPA, positive & $0.95(0.66 ; 1.35)$ & 0.76 & & \\
\hline Erosions, present at BL & $0.80(0.49 ; 1.29)$ & 0.35 & & \\
\hline
\end{tabular}

ACPA: anti-citrullinated protein antibodies; BL: baseline; DAS: disease activity score; RF: rheumatoid factor. ${ }^{\alpha}$ The final multivariable logistic regression model was based on stepwise for ward and backward selection of predictors, both resulting in the same final model.

Acknowledgements: We would like to thank all patients for their contribution as well as the rheumatologists who participated in the BeSt study group and in the IMPROVED-study group. We would also like to thank all other rheumatologists 